\title{
Rat Intestinal Acetic Acid and Butyric acid and Effects of Age, Sex, and High-fat Diet on the Intestinal Levels in Rats
}

ORIGINAL

ARTICLE

\author{
Soo In Choi ${ }^{1}$, Nayoung Kim ${ }^{1,2}$, Sun Min Lee ${ }^{1}$, Ryoung Hee Nam¹, So Ra Kang ${ }^{1}$, Chin-Hee Song ${ }^{1}$, Young-Tae Park, \\ Huitae $\mathrm{Min}^{3}$, Yeon-Ran $\mathrm{Kim}^{3}$, Yeong-Jae Seok \\ ${ }^{1}$ Department of Internal Medicine, Seoul National University Bundang Hospital, Seongnam, ${ }^{2}$ Department of Internal Medicine and Liver Research \\ Institute, Seoul National University College of Medicine, ${ }^{3}$ Department of Biological Sciences and Institute of Microbiology, Seoul National University, \\ Seoul, ${ }^{4}$ Korea Institute of Science and Technology Natural Products Research Institute, Gangneung, Korea
}

\begin{abstract}
Background: High-fat diet is known to be implicated in the pathogenesis of various metabolic disorders related to an inflammatory response. The aim of this study was to investigate the influence of high-fat diet for intestinal acetic acid and butyric acid concentrations which are related to inflammation-associated colon cancer risk.

Methods: Both male and female rats of 6, 31, 74 and 104-week of age were fed chow diet or high-fat diet for 8 weeks. Body weight and food intake were measured weekly during the feeding period. Intestinal acetic acid and butyric acid levels were measured by high-performance liquid chromatography from luminal contents of ileum and cecum.

Results: Male rats showed greater weight change than female rats in every age. Calorie-adjusted food intake was also higher in male rats compared to female rats. Male rats showed similar intake of food in every age while 31-week old female rats showed increased intake, which was decreased at 74-week and 104-week of age. The ileal acetic acid concentration was increased in male rats fed high-fat diet, while female rats fed high-fat diet showed no significant change in the ileal acetic acid level. On the other hand, butyric acid almost disappeared in high-fat diet fed rats regardless of sex.

Conclusions: High-fat diet increases the intestinal acetic acid concentration while reducing the butyric acid concentration which may account for increased risk of inflammation-associated colon cancer.
\end{abstract}

(J Cancer Prev 2019;24:20-25)

Key Words: Acetic acid, Butyric acid, Rats, High-fat diets

\section{INTRODUCTION}

High-fat diet is known as a risk factor for various disorders. These include diabetes, cardiovascular disease, and cancer as well as obesity. As a consequence of ingestion of high-fat containing foods, inflammatory responses occur not only in digestive organ tissues such as the liver, intestine, and adipose tissue, but also in the central nervous system including the hypothalamus [1]. The alteration of gut microbiota such as decreased bacterial richness and diversity due to intake of high-fat diet has been investigated extensively in the context of development of chronic systemic inflammation [2,3].

Gut microbiota inhabits along the gastrointestinal tract interacts with host gut environment [4]. Microbes settle in a specific location of the gastrointestinal tract and produce various metabolites, such as short-chain fatty acids (SCFAs) which influence host physiology [5]. SCFAs are produced from the fermentation of dietary fiber which is not degraded and absorbed at small intestine mainly at cecum, the major habitat of gut microbiota [6]. As SCFAs are absorbed through exchange with bicarbonates, its concentration change between the ileum to the cecum affects luminal $\mathrm{pH}$ causing gut microbiota changes [7].

Received March 21, 2019, Revised March 22, 2019, Accepted March 22, 2019

Correspondence to: Nayoung Kim

E-mail: nayoungkim49@empas.com, ORCID: Nayoung Kim, https://orcid.org/0000-0002-9397-0406

Copyright (c) 2019 Korean Society of Cancer Prevention

(c) This is an Open Access article distributed under the terms of the Creative Commons Attribution Non-Commercial License (http://creativecommons.org/licenses/by-nc/4.0) which permits unrestricted non-commercial use, distribution, and reproduction in any medium, provided the original work is properly cited. 
Acetic acid (C2), propionic acid (C3), and butyric acid (C4) are the most important SCFAs derived from microbial fermentative end products in the human colon due to their anti-inflammatory and anti-proliferative properties. Such properties are considered the link between gut microbiota and colon cancer risk [8-10].

Old age is one of the strong risk factors for colon cancer, with $82.4 \%$ of diagnoses occurring at 55 years of age or older and $62 \%$ occurring at 65 years of age or older, according to the age distribution of incidence from 2010 to 2014 reported by the SEER (Surveillance, Epidemiology, and End Results) Program of the National Cancer Institute in US [11]. In addition, sex plays a role during colon inflammation and immune response due to different gene and sex hormone levels [12,13]. These sexdependent differences have been presumed to result from differences in the concentration of sex hormones, expression of genes on $\mathrm{X}$ and $\mathrm{Y}$ chromosomes, and percentage of body fat [14].

From this background, we investigated the association of high-fat diet with colon cancer with focus on its impact on gut microbiota. Also, we conducted experiments with both male and female rats of various ages (young to old age) to investigate the effects of sex and age on high-fat diet-induced colon cancer.

\section{MATERIALS AND METHODS}

\section{Animals and sample collection}

Specific-pathogen-free (SPF) F344/NSIc rats of 6, 31, 74, and 104-week of age with both sexes were used (Orient, Seoul, Korea). The rats were kept under SPF conditions at $23^{\circ} \mathrm{C}$ and under 12:12-hour light-dark cycles. Rats were divided into two different commercial diets: chow and high-fat diets (chow: $3.85 \mathrm{kcal} / \mathrm{g}$;
A

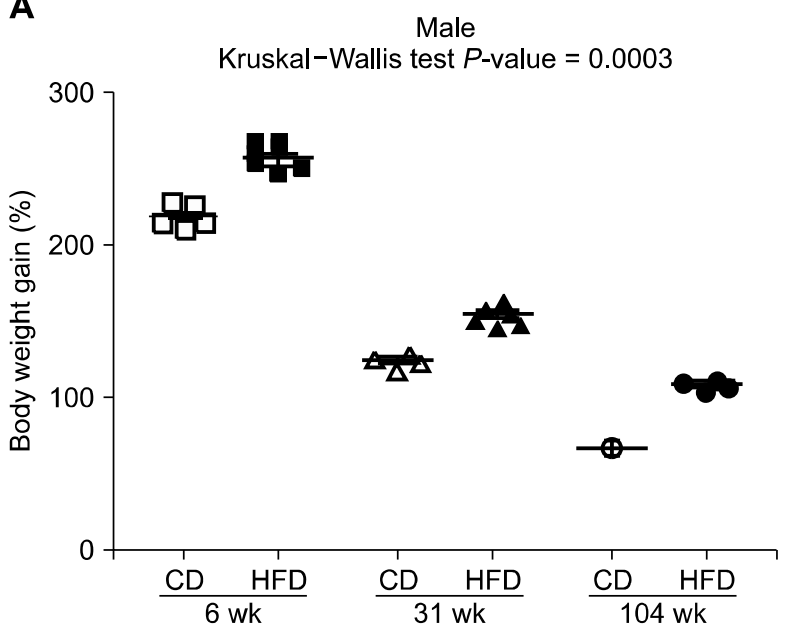

B
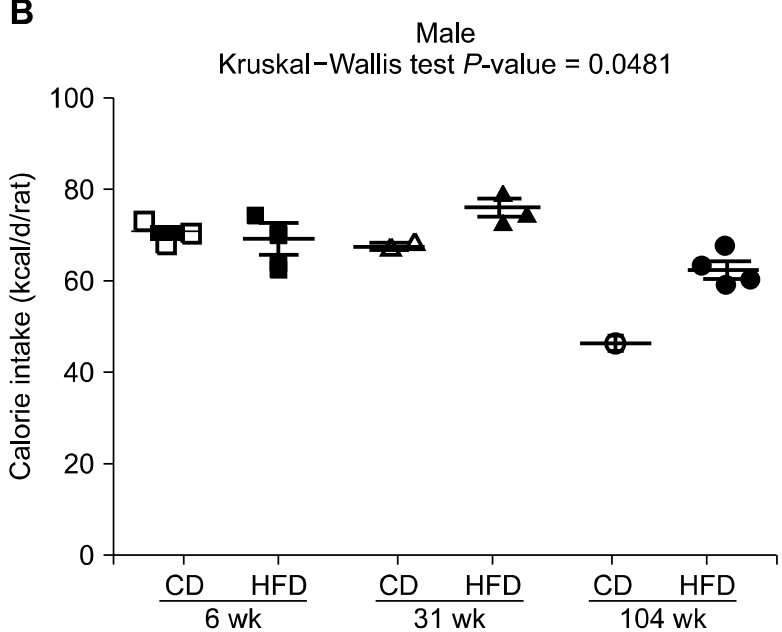

Female

Kruskal-Wallis test $P$-value $<0.0001$

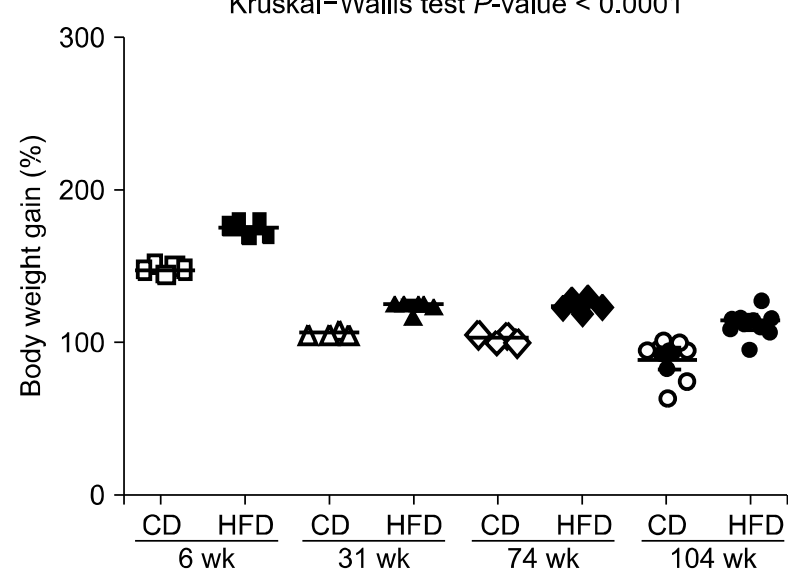

Female

Kruskal - Wallis test $P$-value $=0.0097$

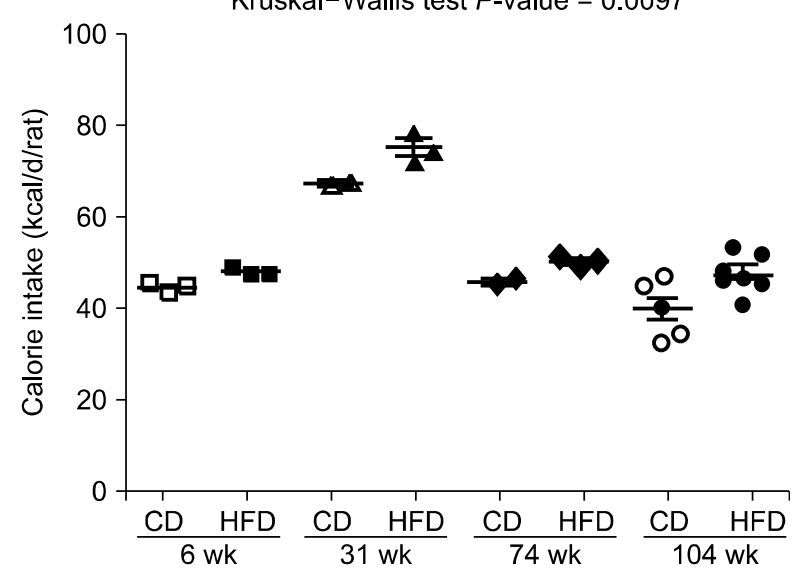

Figure 1. Body weight gain (\%) (A) and calorie intake (kcal/d/rat) (B) at the end of the 8-week feeding period compared with the start of the period. Calorie intake adjusted from daily food intake of each food. Data are expressed as mean \pm SEM; $P$-values to Kruskal-Wallis test are designated on the figure. $\mathrm{CD}$, chow diet; HFD, high-fat diet. 
high-fat: $5.24 \mathrm{kcal} / \mathrm{g}$, $60 \%$ of calorie from fat) and were fed ad libitum. Each diet was provided by Research Diets, Inc. (New Brunswick, NJ, USA). Consequently, there were 14 groups depending on the different ages, sexes, and diets: Group 1, 6-week-old male chow $(\mathrm{n}=8$ ); Group 2, 6-week-old male high-fat $(\mathrm{n}=5)$; Group 3, 31-week-old male chow ( $\mathrm{n}=7$ ); Group 4 , 31-week-old male high-fat $(n=6)$; Group 5, 104-week-old male chow ( $n=5)$; Group 6, 104-week-old male high-fat $(n=4)$; Group 7, 6-week-old female chow ( $n=8$ ); Group 8, 6-week-old female high-fat $(\mathrm{n}=5)$; Group 9, 31-week-old female chow $(\mathrm{n}=6)$ ); Group 10, 31-week-old female high-fat $(\mathrm{n}=6)$; Group 11, 74-week-old female chow ( $\mathrm{n}=5$ ); Group 12, 74-week-old female high-fat ( $\mathrm{n}=$ 6); Group 13, 104-week-old female chow ( $n=10$ ); Group 14 , 104-week-old female high-fat $(n=10)$. The 74-week-old male group was excluded because of natural death due to senescence. During the 8 weeks of feeding high-fat diets, the body weight and food intake of each rat were measured weekly. After the 8-week feeding period, ileum and cecum tissue specimens with luminal contents were collected for fatty acid analysis. Terminal anesthesia was conducted via inhalation of carbon dioxide.

This study was performed in accordance with the recommendations of the Guide for the Care and Use of Laboratory Animals of South Korea. The protocol was approved by the Institutional Animal Care and Use Committee of the Seoul National University Bundang Hospital (permission number: BA1304-127/033).

\section{Measurements of acetic acids and butyric acids concentrations}

Contents of ileum and cecum were extracted as described previously [15] and the concentrations of acetic acid and butyric acid in each extract were measured via high-performance liquid chromatography. Briefly, distilled water was added to 20 to $50 \mathrm{mg}$ of each intestinal contents to a final weight of $200 \mathrm{mg}$. Following incubation at $80^{\circ} \mathrm{C}$ for 15 minutes, each sample was centrifuged at $13,000 \mathrm{rpm}$ for 10 minutes, after which the supernatant was filtered through a membrane filter (pore size $0.45 \mu \mathrm{m}$ ). Acetic acid and butyric acid in each sample were then separated and measured using an Agilent 1100 series instrument (Agilent, Santa Clara, CA, USA) equipped with a C18 column (ZORBAX Eclipse XDB-C18, analytical $4.6 \times 150 \mathrm{~mm}$, 5-Micron; Agilent) and a UV detector $(210 \mathrm{~nm})$. The mobile phase consisted of $90 \% 10 \mathrm{mM}$ $\mathrm{KH}_{2} \mathrm{PO}_{4}$ and $10 \%$ acetonitrile.

\section{Statistical analysis}

Data are expressed as mean \pm SEM. Statistical analyses were performed using PASW Statistics ver. 18.0 (IBM Co., Armonk, NY, USA). Statistical significance was examined with the KruskalWallis test or Mann-Whitney U-test with Bonferroni correction. A $P$-value of less than 0.05 was considered statistical significance.

\section{RESULTS}

Firstly, daily food intake adjusted as calorie intake was

B

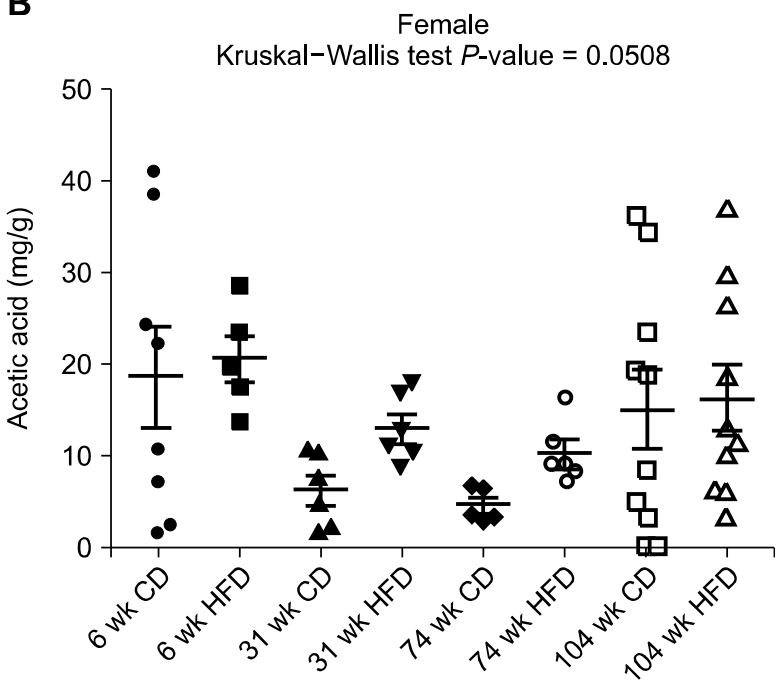

Figure 2. Ileal acetic acid concentration $(\mathrm{mg} / \mathrm{g})$ of male (A) and female (B) rats. Data are expressed as mean \pm SEM; $P$-values to Kruskal-Wallis test are designated on the figure; ${ }^{*} P<0.05 ;{ }^{*} P<0.01$; ns: not significant; representing statistical significance according to Mann-Whitney U-test with Bonferroni correction. CD, chow diet; HFD, high-fat diet. 
compared together with body weight change. All 14 groups gained weight compared with the start point of the 8-week feeding period and the end of the period. However, the ratio of weight gain was decreased through the age in both sexes (Fig. 1A). Also, the high-fat fed group showed greater weight gain compared to chow fed group in the same age and sex. Calorie intake was generally high in male rats compared with females (Fig. 1B). In the case of the 31-week-old female group, there was an increased calorie intake even similar to the 31-week-old male group.

The acetic acid concentration was measured in ileal and cecal luminal contents (Fig. 2, Table 1). All 14 groups showed a reduced acetic acid concentration in cecum compared with ileum, except for the 104-week-old male chow group. In the case of ileum, the high-fat diet group showed an elevated acetic acid concentration trends compared to the chow diet group, especially statistically significant elevation in the 6-week-old male and, 104-week-old male group (Fig. 2A). For the cecal acetic acid concentration, there was no significant difference by diet, while both male and female middle age groups (31-week-old and 74-week-old) showed a decreased acetic acid concentration compared with that in young (6-week-old) and old (104-week-old) age group groups (Table 1).

The butyric acid concentrations in ileal and cecal luminal

Table 1. Acetic acid concentration of ileal and cecal lumen contents

\begin{tabular}{|c|c|c|c|c|c|c|c|}
\hline \multirow{2}{*}{ Sex } & \multirow{2}{*}{ Age (wk) } & \multirow{2}{*}{ Diet } & \multirow{2}{*}{$\begin{array}{l}\text { Number of } \\
\text { samples }\end{array}$} & \multicolumn{2}{|c|}{ Ileum } & \multicolumn{2}{|c|}{ Cecum } \\
\hline & & & & Mean (mg/g) & SEM & Mean (mg/g) & SEM \\
\hline \multirow[t]{6}{*}{ Male } & 6 & Chow & 8 & 8.42 & 2.82 & 4.53 & 0.82 \\
\hline & & High-fat & 5 & 27.13 & 4.26 & 5.60 & 0.87 \\
\hline & 31 & Chow & 7 & 6.92 & 2.14 & 2.76 & 0.96 \\
\hline & & High-fat & 6 & 9.28 & 1.92 & 0.46 & 0.10 \\
\hline & 104 & Chow & 5 & 9.77 & 6.84 & 9.88 & 2.87 \\
\hline & & High-fat & 4 & 17.66 & 2.86 & 7.45 & 0.57 \\
\hline \multirow[t]{8}{*}{ Female } & 6 & Chow & 8 & 18.58 & 5.54 & 9.07 & 2.65 \\
\hline & & High-fat & 5 & 20.60 & 2.53 & 6.82 & 1.52 \\
\hline & 31 & Chow & 6 & 6.35 & 1.60 & 0.41 & 0.05 \\
\hline & & High-fat & 6 & 12.95 & 1.53 & 0.45 & 0.09 \\
\hline & 74 & Chow & 5 & 4.70 & 0.81 & 0.66 & 0.18 \\
\hline & & High-fat & 6 & 10.36 & 1.37 & 1.11 & 0.44 \\
\hline & 104 & Chow & 10 & 15.05 & 4.33 & 4.64 & 1.55 \\
\hline & & High-fat & 10 & 16.30 & 3.61 & 3.73 & 1.18 \\
\hline
\end{tabular}

A

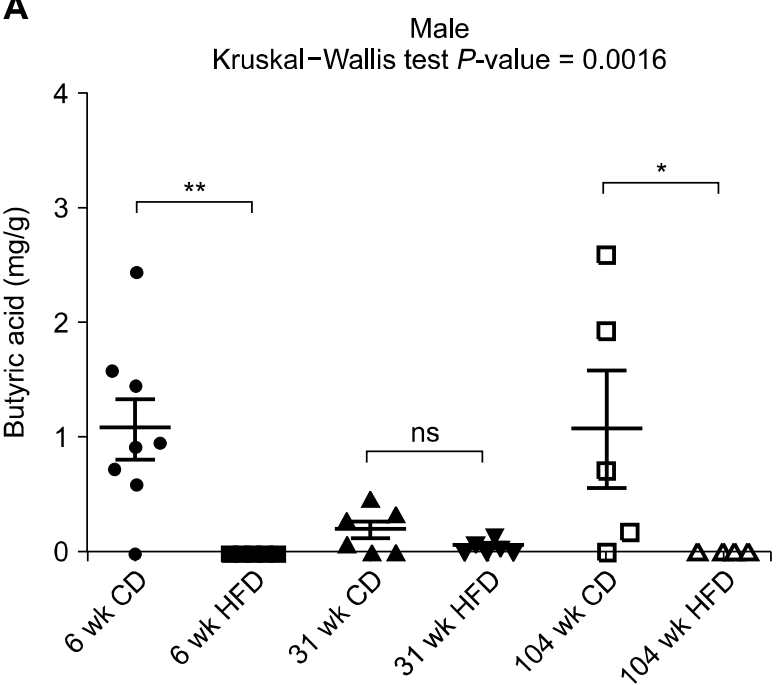

B

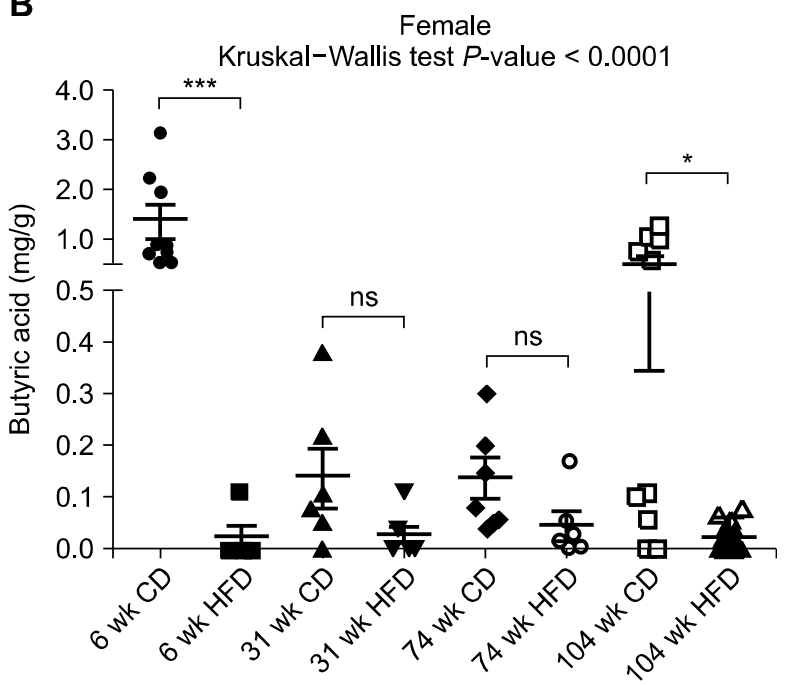

Figure 3. Cecal butyric acid concentration (mg/g) of male (A) and female (B) rats. Data are expressed as mean \pm SEM; $P$-values to Kruskal-Wallis test are designated on the figure; $* P<0.05 ; * * P<0.01 ; * * * P<0.001$; ns: not significant; considered as statistical significance according to Mann-Whitney U-test with Bonferroni correction. CD, chow diet; HFD, high-fat diet. 
Table 2. Butyric acid concentration of ileal and cecal lumen contents

\begin{tabular}{|c|c|c|c|c|c|c|c|}
\hline \multirow{2}{*}{ Sex } & \multirow{2}{*}{ Age (wk) } & \multirow{2}{*}{ Diet } & \multirow{2}{*}{$\begin{array}{l}\text { Number of } \\
\text { samples }\end{array}$} & \multicolumn{2}{|c|}{ Ileum } & \multicolumn{2}{|c|}{ Cecum } \\
\hline & & & & Mean $(\mathrm{mg} / \mathrm{g})$ & SEM & Mean (mg/g) & SEM \\
\hline \multirow[t]{6}{*}{ Male } & 6 & Chow & 8 & 0.20 & 0.06 & 1.08 & 0.26 \\
\hline & & High-fat & 5 & 0.00 & 0.00 & 0.00 & 0.00 \\
\hline & 31 & Chow & 7 & 0.10 & 0.05 & 0.21 & 0.07 \\
\hline & & High-fat & 6 & 0.04 & 0.02 & 0.05 & 0.02 \\
\hline & 104 & Chow & 5 & 0.54 & 0.35 & 1.08 & 0.50 \\
\hline & & High-fat & 4 & 0.00 & 0.00 & 0.00 & 0.00 \\
\hline \multirow[t]{8}{*}{ Female } & 6 & Chow & 8 & 0.20 & 0.10 & 1.38 & 0.34 \\
\hline & & High-fat & 5 & 0.00 & 0.00 & 0.02 & 0.02 \\
\hline & 31 & Chow & 6 & 0.06 & 0.05 & 0.14 & 0.06 \\
\hline & & High-fat & 6 & 0.00 & 0.00 & 0.03 & 0.02 \\
\hline & 74 & Chow & 5 & 0.00 & 0.00 & 0.14 & 0.04 \\
\hline & & High-fat & 6 & 0.00 & 0.00 & 0.05 & 0.03 \\
\hline & 104 & Chow & 10 & 0.29 & 0.21 & 0.51 & 0.16 \\
\hline & & High-fat & 10 & 0.01 & 0.01 & 0.02 & 0.01 \\
\hline
\end{tabular}

contents were also assessed (Fig. 3, Table 2). In the case of high-fat fed group, butyric acid was barely detected. In the chow fed group, there was at least two-fold increase in the butyric acid concentration in cecum compared with ileum. Similar to acetic acid, both in ileum and cecum, the concentration of butyric acid decreased at middle age (31-week-old and 74-week-old) and comparatively high in young (6-week-old) and old (104-week-old) group in both sexes (Table 2).

\section{DISCUSSION}

In this our study, we observed the different response to high-fat ingestion which was somehow related to sex and various age range (young, middle and old age range). Reduction of the acetic acid concentration in cecum compared with ileum may be attributable to absorption of acetic acid that the luminal contents moves to cecum [7]. Moreover, increment of the acetic acid concentration under high-fat diet condition may be due to decreased cecal absorption ability in order to inhibit excessive energy intake compared with the chow group [16,17]. The cecal acetic acid concentration reduction in middle age compared with young and old age may implicate high capacity of digestive ability of youth [18].

Significant reduction of butyric acid concentration under high-fat diet condition regardless of age, sex, or intestinal location strongly suggests harmful impact of high-fat diet on gut microbiota and intestinal tissues. The reduction of butyric acid concentration under disease condition has been reported [19,20]. The most investigated mechanism of tumor suppressive function of butyric acid is inhibition of histone deacetylases implicated in many oncogenic pathways [21]. However, lower doses (about 0.02 $\mathrm{mg} / \mathrm{g}$ ) of butyric acid have a differential effect on cell proliferation depending on the Warburg effect, provoking hyper-proliferative condition. On the other hands, high doses (about $0.2 \mathrm{mg} / \mathrm{g}$ ) of butyric acid were shown to inhibit proliferation regardless of the Warburg effect [22]. In our study, dramatic extinction of butyric acid at cecum under high-fat fed group $(0-0.05 \mathrm{mg} / \mathrm{g})$ compared to chow fed group (0.14-1.38 mg/g) also suggests increased colon cancer risk of high-fat diet.

The measurement of SCFAs concentration of intestine is hard to be a direct evidence of gut microbial dysbiosis or inflammatory response of intestinal epithelial tissue due to high-fat diet. However, a previous study showed the alteration of gut microbiota by high-fat diet in old rat [23,24]. Further study will be necessary to inflammatory response followed by SCFAs concentration changes depend on the gastrointestinal location by high-fat diet.

In conclusion, the acetic acid and butyric acid concentrations are influenced by diet, sex, and age, which implicate different colon cancer risk of different featured groups.

\section{ACKNOWLEDGMENTS}

This work was supported by the National Research Foundation (NRF) of Korea funded by the Ministry of Science, ICT and Future Planning (No. NRF-2016R1A2B4013133).

\section{CONFLICTS OF INTEREST}

No potential conflicts of interest were disclosed. 


\section{REFERENCES}

1. Duan Y, Zeng L, Zheng C, Song B, Li F, Kong X, et al. Inflammatory links between high fat diets and diseases. Front Immunol 2018:9:2649.

2. Murphy EA, Velazquez KT, Herbert KM. Influence of high-fat diet on gut microbiota: a driving force for chronic disease risk. Curr Opin Clin Nutr Metab Care 2015;18:515-20.

3. He C, Cheng D, Peng C, Li Y, Zhu Y, Lu N. High-fat diet induces dysbiosis of gastric microbiota prior to gut microbiota in association with metabolic disorders in mice. Front Microbiol 2018; 9:639.

4. Li D, Chen H, Mao B, Yang Q, Zhao J, Gu Z, et al. Microbial biogeography and core microbiota of the rat digestive tract. Sci Rep 2017:8:45840

5. Brownawell AM, Caers W, Gibson GR, Kendall CW, Lewis KD, Ringel Y, et al. Prebiotics and the health benefits of fiber: current regulatory status, future research, and goals. J Nutr 2012;142: 962-74.

6. Miller TL, Wolin MJ. Pathways of acetate, propionate, and butyrate formation by the human fecal microbial flora. Appl Environ Microbiol 1996;62:1589-92.

7. den Besten G, van Eunen K, Groen AK, Venema K, Reijngoud DJ, Bakker BM. The role of short-chain fatty acids in the interplay between diet, gut microbiota, and host energy metabolism. J Lipid Res 2013:54:2325-40.

8. Ou J, Carbonero F, Zoetendal EG, DeLany JP, Wang M, Newton K, et al. Diet, microbiota, and microbial metabolites in colon cancer risk in rural Africans and African Americans. Am J Clin Nutr 2013;98:111-20.

9. Rivière A, Selak M, Lantin D, Leroy F, De Vuyst L. Bifidobacteria and butyrate-producing colon bacteria: importance and strategies for their stimulation in the human gut. Front Microbiol 2016; 7:979.

10. Louis P, Hold GL, Flint HJ. The gut microbiota, bacterial metabolites and colorectal cancer. Nat Rev Microbiol 2014;12:661-72.

11. Noone AM, Howlader N, Krapcho M, Miller D, Brest A, Yu M, et al. SEER Cancer Statistics Review (CSR) 1975-2015. National Cancer Institute. https://seer.cancer.gov/csr/1975_2015/.

12. Klein SL, Flanagan KL. Sex differences in immune responses. Nat
Rev Immunol 2016;16:626-38.

13. Kim SE, Paik HY, Yoon H, Lee JE, Kim N, Sung MK. Sex- and gender-specific disparities in colorectal cancer risk. World J Gastroenterol 2015;21:5167-75.

14. Regitz-Zagrosek V. Why do we need gender medicine? In: Oertelt-Prigione S, Regitz-Zagrosek V, eds. Sex and Gender Aspects in Clinical Medicine. London, Springer, pp 1-4, 2012.

15. Mishiro T, Kusunoki R, Otani A, Ansary MM, Tongu M, Harashima N, et al. Butyric acid attenuates intestinal inflammation in murine DSS-induced colitis model via milk fat globule-EGF factor 8. Lab Invest 2013;93:834-43.

16. Schwiertz A, Taras D, Schäfer K, Beijer S, Bos NA, Donus C, et al. Microbiota and SCFA in lean and overweight healthy subjects. Obesity (Silver Spring) 2010;18:190-5.

17. Patil DP, Dhotre DP, Chavan SG, Sultan A, Jain DS, Lanjekar VB, et al. Molecular analysis of gut microbiota in obesity among Indian individuals. J Biosci 2012;37:647-57.

18. Varga F. Transit time changes with age in the gastrointestinal tract of the rat. Digestion 1976;14:319-24.

19. Jakobsdottir G, Xu J, Molin G, Ahrné S, Nyman M. High-fat diet reduces the formation of butyrate, but increases succinate, inflammation, liver fat and cholesterol in rats, while dietary fibre counteracts these effects. PLoS One 2013;8:e80476.

20. Huda-Faujan N, Abdulamir AS, Fatimah AB, Anas OM, Shuhaimi $\mathrm{M}$, Yazid AM, et al. The impact of the level of the intestinal short chain Fatty acids in inflammatory bowel disease patients versus healthy subjects. Open Biochem J 2010;4:53-8.

21. Chen J, Vitetta L. Inflammation-modulating effect of butyrate in the prevention of colon cancer by dietary fiber. Clin Colorectal Cancer 2018;17:e541-4.

22. Donohoe DR, Collins LB, Wali A, Bigler R, Sun W, Bultman SJ. The Warburg effect dictates the mechanism of butyrate-mediated histone acetylation and cell proliferation. Mol Cell 2012;48: 612-26.

23. Lee SM, Kim N, Yoon H, Nam RH, Lee DH. Microbial changes and host response in F344 rat colon depending on sex and age following a high-fat diet. Front Microbiol 2018;9:2363.

24. Lee SM, Kim N, Park JH, Nam RH, Yoon K, Lee DH. Comparative analysis of ileal and cecal microbiota in aged rats. J Cancer Prev 2018;23:70-6. 